\title{
Vorschlagsliste verordnungsfähiger Arzneimittel (sog. Positivliste) Was bedeutet dies für die Psychopharmakotherapie?
}

\author{
J. Fritze ${ }^{1,2}$, M. Schmauß1, F. Holsboer ${ }^{2}$ \\ 1 für den Vorstand der Deutschen Gesellschaft für Psychiatrie, Psychotherapie und \\ Nervenheilkunde (DGPPN) \\ 2 für den Vorstand der Arbeitsgemeinschaft für Neuropsychopharmakologie und \\ Pharmakopsychiatrie (AGNP)
}

G emäß § 33 a SGB V (Verordnungsfähige Arzneimittel) ist das Bundesministerium für Gesundheit und Soziale Sicherung (BMGS) ermächtigt, durch Rechtsverordnung mit Zustimmung des Bundesrates auf der Grundlage einer Vorschlagsliste eine Liste verordnungsfähiger Arzneimittel unter Berücksichtigung der Indikationen und Darreichungsformen in der vertragsärztlichen Versorgung zu erlassen. Ein Entwurf der Vorschlagsliste sei am 13.07.2001 den in § 33a Abs. 9 SGB V genannten Fachgesellschaften, Verbänden und Vereinigungen zur Kenntnis gebracht worden. Am 15.11.2002 wurde nun die am 26.04.2002 vom Institut dem BMGS übergebene Vorschlagsliste den Anhörungsberechtigten zugeleitet; die Anhörungsfrist endete bereits am 10.12.2002.

Bundesgesundheits- und -sozialministerin Ulla Schmidt hat den Entwurf eines Gesetzes über die Verordnungsfähigkeit von Arzneimitteln in der vertragsärztlichen Versorgung (Arzneimittel-Positivlistengesetz - AMPoLG) eingebracht. Obwohl § 33a SGB V die Zustimmung des Bundesrates vorschreibt, strebt der Gesetzentwurf an, die Zustimmungspflicht des Bundesrates zu erübrigen.

Aus unerfindlichen Gründen gehörte die AGNP weder zu den Empfängern des Entwurfes der Vorschlagsliste noch der endgültigen Vorschlagsliste, konnte also nicht Stellung nehmen. Die AGNP behält sich angesichts des widerrechtlichen Ausschlusses vom Anhörungsverfahren rechtliche Schritte vor.
Die DGPPN hatte ihre Unterstützung gemäß § 33a SGB V Abs. 8 verbunden mit dem Hinweis auf grundlegende Bedenken gegen eine sog. Positivliste wie auch gegen das Verfahren, mit dem die Positivlistenkommission eingerichtet und die Positivliste entwickelt werden sollte. Die DGPPN und die Arbeitsgemeinschaft für Neuropsychopharmakologie und Pharmakopsychiatrie (AGNP) haben gemeinsam diese Bedenken im Rahmen des Gesetzgebungsverfahrens mündlich und schriftlich vorgetragen und publiziert (Nervenarzt 70 (1999) 766-771 und Psycho 25 (1999) 96-101). Die DGPPN hat auch im Nachgang zum Gesetzgebungsverfahren kritisch dazu Stellung genommen (Nervenarzt 71 (2000) 225-226, Psycho 26 (2000) 108-109). Die Kritik richtet sich i.w. dagegen, dass den regulären, inzwischen auf europäischer Ebene agierenden Zulassungsbehörden, denen eine adäquate Infrastruktur für ordentliches wissenschaftliches Arbeiten zur Verfügung steht, auf nationaler Ebene letztlich eine Instanz übergeordnet wird, der diese wissenschaftliche Infrastruktur fehlt.

Die Vorschlagsliste wurde unter Geschäftsführung durch Herrn Prof. Dr. Klaus Quiring in ehrenamtlicher Tätigkeit unter dem Vorsitz von Prof. Dr. Ulrich Schwabe (Heidelberg) durch die Herren Prof. Dr. Johannes Köbberling (Wuppertal), Prof. Dr. Adalbert Keseberg (Erftstadt-Liblar), Dr. Jürgen Bausch (Frankfurt am Main) (Stellvertreterin: Frau Dr. Rieke Alten (Berlin)), Prof. Dr. Ulrich Schwabe (Heidelberg), Prof. Dr. Hans Joachim Trampisch (Bochum) (Stell- vertreter: Prof. Dr. Jürgen Windeler (Essen)), Prof. Dr. Reinhard Saller (Zürich) (Stellvertreterin: Frau PD Dr. Karin Kraft (Bonn)), Dr. KarlHeinz Gebhardt (Karlsruhe) (Stellvertreter: Dr. Michael Elies (Laubach)), Dr. Harald Matthes (Berlin) (Stellvertreter: Dr. Markus Karutz (Köln)) und Frau Prof. Dr. Petra Thürmann (Wuppertal) (Stellvertreter: Prof. Dr. Henning Breithaupt (Gießen)) erarbeitet. Gemäß § 33a SGB V Abs. 3 haben die Mitglieder der Kommission unparteilich zu sein. Dies soll nicht in Frage gestellt werden, auch wenn einzelne Mitglieder bzw. Stellvertreter z.B. als Herausgeber des Arzneiverordnungsreports, als frühere Vorsitzende einer kassenärztlichen Vereinigung bzw. als Mitarbeiter des Medizinischen Dienstes der Spitzenverbände der Krankenkassen bekannt sind.

Gemäß § 33a SGB V Abs. 7 sind in die Vorschlagsliste Arzneimittel aufzunehmen, „die für eine zweckmäßige, ausreichende und notwendige Behandlung, Prävention oder Diagnostik von Krankheiten oder erheblichen Gesundheitsstörungen geeignet sind; Voraussetzung für diese Eignung ist ein mehr als geringfügiger therapeutischer Nutzen, gemessen am Ausmaß des erzielbaren therapeutischen Effekts. Den indikationsbezogenen Bewertungen sind jeweils einheitliche Urteilsstandards zugrunde zu legen. In die Bewertungen einzubeziehen sind Qualität und Aussagekraft der Belege, die therapeutische Relevanz der wissenschaftlichen Erkenntnisse und die Erfolgswahrscheinlichkeit der therapeutischen, präventiven 


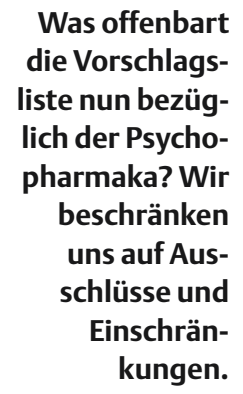

Was offenbart
die Vorschlags-
liste nun bezüg-
lich der Psycho-
pharmaka? Wir
beschränken
uns auf Aus-
schlüsse und
Einschrän-
kungen.

bei Präeklampsie und Eklampsie, wenn Arzneimittel mit einem günstigeren Nutzen/Risiko-Verhältnis nicht ausreichend wirksam bzw. anwendbar sind" nicht mehr genutzt werden. Dasselbe gilt für die - nicht mehr beanspruchte - Indikation „Behandlung von schweren Schlafstörungen in höherem Lebensalter". Letzteres ist angesichts anderer, verträglicherer Alternativen wohl vertretbar, wenn auch ein Verlust.

Chloralhydrat wäre nicht mehr zulasten der GKV verschreibungsfähig. Dies erstaunt zumindest auf den ersten Blick, indem Chloralhydrat auf eine 140-jährige Anwendung zurückblickt (Entdeckung 1831 durch Justus von Liebig; Beobachtung der hypnotischen Wirkung 1861 durch Rudolf Buchheim). Viele Jahre wurde auch in der jüngeren Vergangenheit Chloralhydrat als „escape medication" auch in Zulassungsstudien von Antidepressiva eingesetzt. Seine Tagesbehandlungskosten sind gering $(<0,32$ Euro), der Packungspreis liegt im Bereich der Zuzahlung. Das Abhängigkeitsrisiko ist gering, wenn auch nicht auszuschließen. Ausschlaggebend für den Ausschluss war vermutlich das Fehlen adäquater Studien (die bei einem derart alten Pharmakon kaum zu erwarten sind) sowie der aversive (bittere) Ge- schmack, die gastrointestinalen Nebenwirkungen (Einzelfälle; Chloralhydrat reizt wie alle Aldehyde die Magenschleimhaut), der Geruch der Atemluft nach Aldehyden, die seltenen Nebenwirkungen Nierenschäden, Hautallergien, Leberschäden, Leukopenie, Eosinophilie, und die geringe therapeutische Breite (relevant bei Intoxikation) infolge Kardiotoxizität des aktiven Metaboliten Trichloroethanol und des inaktiven Metaboliten Trichloressigsäure. Leider legt das Institut die Gründe für Ausschlüsse und Einschränkungen nicht dar.

\section{Antidementiva}

Unter den Antidementiva wäre Tacrine nicht mehr verordnungsfähig; dies erscheint angesichts seiner Lebertoxizität und der Verfügbarkeit verträglicherer und sicherer Alternativen (Donepezil, Rivastigmin, Galantamin) gerechtfertigt. Allerdings stellt sich die Frage, ob vor diesem Hintergrund die arzneimittelrechtliche Zulassung von Tacrine überhaupt Bestand haben kann. Memantine und Ginkgo biloba wären nur noch als Antidementivum verordnungsfähig. Der Ausschluss der weiteren Indikationen (depressive Verstimmung, Schwindel, Ohrensausen, Kopfschmerzen) von Ginkgo biloba tangiert die Neuropsychopharmakotherapie nicht bedeutsam.

Schwieriger ist die Lage bei Memantine. Memantine war bis Anfang 2002 fiktiv zugelassen für die Indikationen (sinngemäß) leichte und mittelschwere Hirnleistungsstörungen, aber auch bei Störungen der Motorik wie z. B. bei zentral bedingten Bewegungsstörungen, frühkindlichen Hirnschädigungen, Schädelhirntraumen, Multipler Sklerose, zerebraler Ischämie und parkinsonähnliche Erkrankungen. Im Rahmen seiner europäischen Zulassung wurde die Indikation eingeschränkt auf „Behandlung von Patienten mit mittelschwerer bis schwerer Alzheimer-Krankheit“; als Auflage musste auf den altem Handelsnamen Akatinol ${ }^{\circledR}$ verzichtet werden. Mit dem neuen Handelsnamen wurden die Tagesbehandlungskosten erheblich erhöht (im Interesse der Kompatibilität mit dem internationalen Preis- gefüge des im Gegensatz zu Deutschland in anderen Ländern neu eingeführten Memantine). Mit der Rücknahme des Akatinol ${ }^{\circledR}$ vom Markt stellt die Verordnung von Memantine in den weggefallenen Indikationen Off-Label-Use mit allen damit verbundenen Problemen (siehe Nervenarzt 73 (2002) 796-799; Psycho 28 (2002) 431-439; Nervenarzt 73 (2002) 1210-1212) dar.

Weitere Ausschlüsse von Wirkstoffen, die früher bei unscharfen Indikationen wie Hirnleistungsstörungen eingesetzt wurden, wie Pentoxifyllin, Naftidrofuryl, Cyclandelat, Moxaverin, Diisopropylammonium, Xantinol, Etofyllin, Bencyclan, erscheinen vertretbar angesichts fehlender Wirksamkeitsbelege.

Dies gilt auch für die Nootropika im engeren Sinne wie Pirazetam, Nicergolin, Meclofenoxat, Vinpocetin, Pyritinol, Nimodipin (nur bezüglich Demenz), Cinnarizin (bezüglich Demenz; verordnungsfähig zur Migräneprophylaxe), Dihydroergotoxin. Das Institut folgt hier den Bewertungen durch die Arzneimittelkommission der deutschen Ärzteschaft (AkdÄ). Hintergrund sind die methodischen Mängel der - überwiegend vor vielen Jahren durchgeführten - Studien. Institut und AkdÄ ignorieren dabei aber, dass Vergleichsstudien zu Cholinesterasehemmern (Donepezil, Rivastigmin, Galantamin) nie durchgeführt wurden (und vermutlich niemals durchgeführt werden), sodass der behauptete geringfügige Nutzen (als mutmaßlicher Ausschlussgrund) der Nootropika (bei günstigen Tagesbehandlungskosten) niemals bewiesen werden wird.

Unter den Neuroleptika kann Promethazin (eigentlich kein Neuroleptikum, u.a. da ohne Affinität zu Dopaminrezeptoren) nur noch als Hypnotikum und Sedativum (nicht als Antiallergikum) verordnet werden. Triflupromazin darf nicht als Antiemetikum eingesetzt werden (was angesichts der extrapyramidalmotorischen Nebenwirkungen akzeptabel ist). Trifluperazin wird ohnehin nicht mehr vertrieben, sodass es in die Liste nicht aufgenommen werden konnte. Dasselbe gilt für Triperidol und Sertindol. 
Das nicht neuroleptisch wirksame Tiaprid, zugelassen für die Indikationen „Dyskinesien, z.B. dystone Syndrome wie Torsionsdystonien, Hemiballismus, Tics, iatrogene Dyskinesien nach Gabe von L-Dopahaltigen Arzneimitteln, Dyskinesien nach Neuroleptika-Therapie; Bewegungsanomalien, vor allem Chorea und choreatische Zustände, Chorea Huntington" ist ausgeschlossen. Vermutlich wird es häufiger als in diesen zugelassenen Indikationen offlabel eingesetzt im Alkoholentzug, in der Gerontologie und bei verschiedenen psychiatrischen Indikationen (z.B. beim Tourette-Syndrom) im Kindes- und Jugendalter. Der Ausschluss ist angesichts des weitgehenden Fehlens methodisch adäquater Studien (RCTs) nachvollziehbar. Er zeigt aber auch die Grenzen des holzschnittartigen Vorgehens der Kommission, langjährige Erfahrung $(1,5)$ zu ignorieren und allein auf RCTs zu fokussieren. Die Patienten werden den Ausschluß nicht danken.

Die auch in spezifisch psychopharmakologischen Indikationen genutzten Antikonvulsiva wie Carbamazepin, Oxcarbazepin, Valproinsäure, Lamotrigin stehen uneingeschränkt zur Verfügung, wobei sich die Kommission selbstverständlich nicht zum Off-label-Use äußert.

Unter den Antidepressiva ist Amitriptylinoxid ausgeschlossen. Dies ist zwar angesichts des weitgehenden Fehlens adäquater Studien nachvollziehbar, medizinisch und pharmakologisch (Amitriptylinoxid ist Prodrug für Amitriptylin) aber unverständlich - Schicksal einer Altsubstanz. Der Psychopharmakotherapie ginge damit eine wahrscheinlich subjektiv verträglichere Alternative (6) zu Amitriptylin verloren. Dasselbe gilt für Lofepramin, Prodrug für Desipramin, das in anderen Ländern (im Gegensatz zu Deutschland) traditionell und unverändert im Antidepressiva-Markt eine bedeutsame Rolle spielt und immerhin in einem RCT dem Paroxetin ebenbürtig war $(2,3)$. Die bisher geringe Nutzung von Lofepramin in Deutschland ist auch deshalb unverständlich, weil Lofepramin ein im Vergleich zu anderen trizyklischen
Antidepressiva geringeres vitales Risiko bei (suizidal motivierter) Intoxikation aufweist (4), was auf der selbstinduzierten Resorptionshemmung infolge Bezoarbildung beruht. Die jeweiligen aktiven Metaboliten bleiben verfügbar.

Das Antidepressivum Bupropion (Amfebutamon; Zyban ${ }^{\circledR}$ ) ist in Europa nur „zur Hilfe bei der Raucherentwöhnung nikotinabhängiger Patienten in Verbindung mit unterstützenden motivierenden Maßnahmen" zugelassen; sein Ausschluss in der Vorschlagsliste entspricht den Arzneimittelrichtlinien, wobei die im Entwurf zur Vorschlagsliste gegebene Begründung „für geringfügige Gesundheitsstörungen bestimmt" zumindest fragwürdig ist: Die Weltgesundheitsorganisation sieht das Rauchen als weltweit wesentlichsten Risikofaktor der Zukunft. Auf das Problem des Off-Label-Use von Bupropion insbesondere bei bipolarer Depression wurde anderenorts eingegangen (Nervenarzt 73 (2002) 1125-1127; Psycho 28 (2002) 597-599).

Naltrexon ist zur Unterstützung bei der psychotherapeutisch/psychologisch geführten Entwöhnungsbehandlung vormals Opiat-Abhängiger nach erfolgter Opiat-Entgiftung zugelassen. Ihm wird in der Vorschlagsliste die Indikation „Mittel zur Behandlung der Alkoholabhängigkeit" zuerkannt; diese Indikation wird zwar wissenschaftlich untersucht, ist aber in Deutschland noch nicht anerkannt. Erfreulich ist, dass Acamprosat - entsprechend den Arzneimittelrichtlinien - in der Vorschlagsliste gemäß zugelassener Indikation „zur Unterstützung der Aufrechterhaltung der Abstinenz beim alkoholabhängigen Patienten im Rahmen eines therapeutischen Gesamtkonzepts, das auch begleitende psycho- und soziotherapeutische Maßnahmen einschließt" enthalten ist.

Unter den Benzodiazepinen und Benzodiazepin-ähnlichen Substanzen ist das ohnehin seit kurzem nicht mehr marktgeführte Clotiazepam ausgeschlossen. Dasselbe gilt für die nicht mehr marktgeführten Substanzen Metaclazepam, Oxazolam und das (nicht-Benzodiazepin)
Meprobamat. Meprobamat ist inadäquat toxisch mit besonders ausgeprägtem Risiko von Dysarthrie, Diplopie und Ataxie. Außerdem besteht die Möglichkeit allergischer Hautreaktionen inkl. Urtikaria sowie von Agranulozytosen. Diese Verluste bedeuten angesichts der Alternativen keinen Mangel. Kavain wurde kürzlich wegen Lebertoxizität vom Markt genommen. Die Hypnotika (Antihistaminika) Doxylamin und Diphenhydramin sind in der Liste enthalten, obwohl sie nicht rezeptpflichtig sind; dies belegt, dass gesetzeskonform - ökonomische Gründe nicht zu Ausschlüssen geführt haben.

\section{a Fazit}

Insgesamt werden die Möglichkeiten der Psychopharmakotherapie durch die Positivliste in ihrer aktuellen Version nicht schwerwiegend eingeschränkt, wenn es auch Kritikpunkte gibt. Auch darf anerkannt werden, dass sich die Zuarbeit der Deutschen Gesellschaft für Psychiatrie, Psychotherapie und Nervenheilkunde (DGPPN) eher gelohnt, jedenfalls nicht geschadet hat. Desto unverständlicher ist, dass die AGNP und DGPPN in den Anhörungsverfahren unberücksichtigt geblieben sind.

\section{Literatur}

1. Dose M, Lange HW. The benzamide tiapride: treatment of extrapyramidal motor and other clinical syndromes. Pharmacopsychiatry 2000; 33: 19-27

2. Kerihuel JC, Dreyfus JF. Meta-analyses of the efficacy and tolerability of the tricyclic antidepressant lofepramine. J Int Med Res 1991; 19: 183-201

3. Moon CA, Vince M.Treatment of major depression in general practice: a doubleblind comparison of paroxetine and lofepramine. Br J Clin Pract 1996; 50: 240-244

4. Reid F, Henry JA.Lofepramine overdosage. Pharmacopsychiatry 1990; 23 Suppl 1: 23-27

5. Roger M, Gerard D, Leger JM. Value of tiapride for agitation in the elderly. Review of published studies. Encephale 1998; 24 : 462-468

6. Tegeler J, Klieser E, Lehmann E, Heinrich K. Double-blind study of the therapeutic efficacy and tolerability of amitriptylinoxide in comparison with amitriptyline. Pharmacopsychiatry $1990 ; 23$ : 45-49

\section{Korrespondenzadresse:}

Prof. Dr. med. J. Fritze

Asternweg 65

D-50259 Pulheim 